\title{
RECURSOS ECONÔMICOS DE IDOSOS EM CORRESIDÊNCIA
}

\author{
ECONOMIC RESOURCES OF THE ELDERLY PEOPLE IN CO-RESIDENCE
}

\author{
Jainne Oliveira Leão Silva ${ }^{1}$, Talita Santos Oliveira Sampaio ${ }^{1}$, Adrielle Santos Souza ${ }^{1}$, Alba \\ Benemérita Alves Vilela ${ }^{2}$, Lucas Silveira Sampaio ${ }^{1}$
}

Faculdade Independente do Nordeste ${ }^{1}$, Universidade Estadual do Sudoeste da Bahia - UESB ${ }^{2}$

\section{Abstract}

The present research had objective to verify eldery's economic resources in situation of cohabitation. This is a descritive, transversal and qualitative research, linked to Eldery Health Research and Studies Center of Faculdade Independente do Nordeste. It were used the Vitória da Conquista's database and included all the eldery cohabitant registered on Health Family Center that had denoted satisfactory condition to answer the questions of Condition Mental Mini Exam, in the total of 239 eldery people. Among the participants, the age found it was between 60 and 95 years old. There was predominance of feminin sex (77\%), marital status married $(42,6 \%)$ and widowed (41,8\%). About the economic aspects it was observed a income predominance of only 1 national minimum wage $(67,4 \%)$ and income source based in retirement income $(87,4 \%)$. It was identified a grater social interaction with grandchildren (57,3\%), sons $(55,2 \%)$ and daughters $(54,0 \%)$. It was verified in this research about the perception of the income to be enough to eldery's basic needs, and always is not suficient. Therefore, this research allows a greater knowledge about this issue, furthering social politics and health for the eldery people and their families, pomoting a better coexistence.

\section{Resumo}

O presente estudo objetivou verificar os recursos econômicos dos idosos em corresidência. Trata-se de um estudo descritivo, transversal $e$ quantitativo, vinculado ao Núcleo de Pesquisa e Estudos em Saúde do Idoso da Faculdade Independente do Nordeste. Utilizou-se o banco de dados do munícipio de Vitória da Conquista e foram incluídos no estudo todos os idosos em corresidência cadastrados nas Unidades de Saúde da Família e que denotaram condições cognitivas de responder aos questionamentos do Mini Exame do Estado Mental, totalizando 239 idosos. Dentre os participantes, a idade encontrada foi entre 60 anos e 95 anos. Houve predominância do sexo feminino (77\%) e do estado civil casado $(42,6 \%)$ e viúvos (41,8\%). Referente aos aspectos econômicos observou-se uma predominância de renda de apenas 1 salário mínimo $(67,4 \%)$ e fonte de renda baseada em aposentadorias e pensões $(87,4 \%)$. Identificou-se uma maior convivência com netos $(57,3 \%)$, seguido de filhos $(55,2 \%) e$ filhas (54,0\%). Verificou-se neste estudo que, quanto a percepção de suficiência de renda para as necessidades básicas dos idosos sempre faltava um pouco. Dessa forma, este estudo permite um maior conhecimento da população $e$ da comunidade cientifica sobre a temática, favorecendo políticas sociais e de saúde para idosos e familiares no intuito de favorecer um bom convívio.
Key words: Elderly; Family Relationships; Income; Family Characteristics.
Palavras chave: Idosos; Relações familiares; Renda familiar; Características da família. 
Introdução

O envelhecimento populacional provoca a formação de novos arranjos familiares, dentre estes, pode-se destacar a corresidência, que se caracteriza pela presença de pelo menos um idoso convivendo com filhos e/ou netos no mesmo espaço domiciliar, essas famílias com idosos podem ser divididas em dois grupos, sendo que no primeiro grupo estão as famílias de idosos, onde este é chefe ou cônjuge e residem com autonomia e no segundo grupo estão as famílias com idosos, onde o idoso mora na condição de parente do chefe, sendo mais vulnerável e que precisa de ajuda dos familiares'.

A corresidência é uma forma de estratégia familiar que beneficia ambos os indivíduos envolvidos ${ }^{2,3}$. Para os idosos, lhes proporcionam segurança, relação próxima com seus descendentes, companheirismo, apoio emocional e cuidados físicos. Em relação aos familiares destaca-se $o$ apoio financeiro advindo dos recursos desse idoso, principalmente quando se encontram em situações como dificuldades em entrar no mercado de trabalho, divórcio e outros problemas econômicos. Além disso, os idosos auxiliam seus filhos em relação aos cuidados com os netos enquanto os pais saem para trabalhar ou procurar emprego 4 .

Em contrapartida, esse convívio pode favorecer o excesso de cuidados por parte da família e privar a capacidade autônoma do idoso, induzindo-o a dependência e também implicando na falta de privacidade e tranquilidade para os mesmos. Além disso, a convivência entre várias gerações pode provocar dificuldades de adaptação, resultando assim em relações conflituosas decorrente das diferenças de crenças, valores sociais e culturais ${ }^{4,5}$.

Sampaio, Sampaio e Vilela ${ }^{3}$ observaram que nos últimos anos o crescimento da corresidência ocorre por maior necessidade dos filhos adultos, seja pela dificuldade em ingressar no mercado de trabalho, problemas financeiros, ou até mesmo quando envolve o processo de divórcio, vendo no idoso uma estabilidade financeira e por isso acabam morando juntos. Dessa forma, grande parte desses idosos continuam a trabalhar para garantir o sustento da família.

Estudos demonstram que uma parcela considerável de idosos, de ambos os sexos, trabalham, representando um dos segmentos sociais com maior estabilidade, sendo responsável na maioria dos casos pela manutenção de suas famílias ${ }^{6}$. Corroborando com esse aspecto, segundo Camarano ${ }^{1}$ o idoso ao se manter no emprego e/ou usufruir do benefício previdenciário, permanece no cargo de provedor da família.

Idosos possuem uma segurança financeira maior e melhor do que os jovens, mesmo que haja gastos imprevistos com medicamentos e tratamentos de saúde. Essa redistribuição intergeracional da renda do idoso faz com que as famílias pobres ou próximas à linha da pobreza que convivem com seus idosos, dependam diretamente da renda destes para obterem melhores condições econômicas ${ }^{6}$. Observa-se que, frequentemente, os idosos corresidentes gastam a maior parte de seu recurso econômico em função de outros familiares e cuidados com a casa, indicando ser uma relação comumente desfavorável, pois se encontram em uma etapa da vida em que deveriam estar investindo a renda no seu bem estar próprio ${ }^{5}$.

Diante disso, a reflexão acerca dos recursos econômicos de idosos em corresidência é urgente e de extrema importância, visto que os idosos são os principais provedores das famílias atualmente, mesmo a grande maioria possuindo apenas aposentadorias e/ou pensões como recurso principal. Ainda, torna-se necessário ampliar o conhecimento sobre o tema uma vez que, mesmo com o aumento da população idosa brasileira e sua nova configuração familiar, a comunidade cientifica ainda dispõe de poucos estudos sobre o conteúdo.

Importante destacar que este estudo poderá contribuir para a criação e a efetivação de políticas públicas sociais e de saúde voltadas para esses idosos e seus familiares. Sendo assim, o objetivo do estudo foi verificar os recursos econômicos dos idosos em corresidência.

\section{Metodologia}

Trata-se de um estudo descritivo, de caráter transversal e abordagem quantitativa. As informações aqui contidas incluídas fazem parte do projeto principal intitulado "Arranjo familiar de idosos residentes em municípios do Nordeste e Sudoeste do Brasil", estando vinculado ao Núcleo de Pesquisa e Estudos em Saúde do Idoso (NUPESI), da Faculdade Independente do Nordeste (FAINOR).

Utilizou-se o banco de dados referente ao munícipio de Vitória da Conquista do estado da Bahia, sendo que das 43 unidades de saúde implantadas no município, segundo o Cadastro Nacional de Estabelecimentos de Saúde, os dados foram coletados em apenas duas Unidades de Saúde da Família (USF) por meio de um sorteio simples.

Foram incluídos no estudo os indivíduos 
com idade igual ou maior que 60 anos residentes na zona urbana do município, que vivessem em situação de corresidência, e que apresentaram condições cognitivas que possibilitasse responder aos questionamentos, sendo a cognição avaliada pelo Mini Exame do Estado Mental - MEEM). O MEEM inclui 11 itens, que demandam respostas verbais a questões de orientação temporal e espacial, atenção, leitura, memória, cálculo, nomeação, seguir comandos verbais e copiar um desenho (polígonos). Para avaliação dos resultados obtidos através do MEEM, adotou-se os seguintes pontos de corte: 19 pontos para idosos analfabetos; 23 pontos para idosos com 1 a 3 anos de escolaridade; 24 pontos para idosos com 4 a 7 anos de escolaridade e 28 pontos para idosos com escolaridade acima de 7 anos. Escores abaixo destas pontuações indicam um risco de déficit cognitivo, requerendo, assim, encaminhamento para avaliação neuropsicológica específica ${ }^{7}$.

Considera-se neste estudo o idoso em estado de corresidência aquele que reside com sua família em uma mesma unidade familiar, podendo conviver uma ou mais gerações, ou seja, filhos e/ou netos e/ou bisnetos dentre outros ${ }^{1}$. Foram excluídos os idosos que apresentaram déficit cognitivo, além dos indivíduos que se recusaram a receber o pesquisador ou não foram encontrados em sua residência após três tentativas em dias e horários alternados. Assim, após a adoção desses critérios de inclusão e exclusão, totalizou-se uma população final de 239 idosos.

A coleta de dados do estudo se baseou no acesso ao banco de dados do projeto principal e transcorreu em duas etapas. Na primeira etapa foi identificado o número de idosos cadastrados nas USF através da consulta das fichas de cadastramento familiar (ficha A) com auxílio dos agentes comunitários de saúde, sendo feita a pesquisa dos idosos que vivem sós e aqueles que corresidem com familiares. A segunda etapa consistiu em aplicar a entrevista nos domicílios dos idosos, por meio de questionário semiestruturado contendo dados de identificação dos participantes, como idade, sexo e composição do arranjo familiar e aplicação do questionário Brazil Old Age Schedule (BOAS).

O BOAS é um instrumento que averigua os fatores multidimensionais do envelhecimento e que foi adaptado, utilizando apenas cinco das oito categorias: Informações gerais do indivíduo, recursos sociais, recursos econômicos, necessidade e problemas que afetam o entrevistado ${ }^{8}$. A equipe que aplicou os instrumentos foi composta por 6 discentes do curso de Fisioterapia da Faculdade Independente do Nordeste - FAINOR, e a coleta de dados aconteceu 5 vezes por semana.

Os dados obtidos através do BOAS, foram tabulados e analisados utilizando o SPSS (versão 21.0). A pesquisa principal foi aprovada pelo Comitê de Ética em Pesquisa da Universidade Estadual do Sudoeste da Bahia (CEP-UESB), cujo parecer $\mathrm{n}$ - 102.641, foi redigido observando a Resolução do Conselho Nacional de Saúde (CNS) no 196/96, vigente naquele período. Sendo assim, foi solicitado a inclusão deste estudo ao projeto principal de acordo a Resolução no 466, de 12 de dezembro de 2012. É relevante destacar que a participação dos idosos foi voluntária e sua aceitação foi formalizada mediante a assinatura do Termo de Consentimento Livre e Esclarecido TCLE, que assinou em duas vias, sendo uma de domínio do informante e a outra da guarda da pesquisadora responsável pelo estudo.

\section{Resultados}

Dentre os idosos participantes, a média de idade encontrada foi de $72,2 \pm 8,08$, com variação mínima de 60 e máxima 95 anos. Verificou-se uma predominância do sexo feminino (77\%) e do estado civil casado $(42,6 \%)$, destaca-se porém, um significativo percentual de viúvos $(41,8 \%)$. No que se refere aos aspectos econômicos observou-se predominância de renda de apenas 01 salário mínimo $(67,4 \%)$ que correspondia ao valor de $\mathrm{R} \$ 954,00$ reais no período da pesquisa e de fonte de renda baseada em aposentadorias e pensões $(87,4 \%)$. Observou-se ainda uma elevada dependência da renda dos filhos (31,4\%). Identificou-se na pesquisa realidade que a maior parcela relatou renda insuficiente para suprir suas necessidades, conforme os dados da Tabela 1.

No que diz respeito ao arranjo familiar da corresidência, identificou-se maior convivência com netos $(57,3 \%)$, seguido de filhos $(55,2 \%)$ e filhas $(54,0 \%)$. (tabela 2$)$. 
Tabela 1. Características sociodemográficas e econômicas de idosos em estado de corresidência. Vitória da Conquista - BA, 2018.

\begin{tabular}{|c|c|c|c|}
\hline Variáveis & \% resposta & $\mathbf{N}$ & $\%$ \\
\hline Sexo & 100 & & \\
\hline Feminino & & 184 & 77,0 \\
\hline Masculino & & 55 & 23,0 \\
\hline Grau de escolaridade & 97,5 & & \\
\hline $\begin{array}{l}\text { Analfabeto } \\
\text { Primário incompleto }\end{array}$ & & $\begin{array}{l}81 \\
108\end{array}$ & $\begin{array}{r}34,8 \\
46,4\end{array}$ \\
\hline Primário completo & & 28 & 12,0 \\
\hline $1^{\circ}$ grau completo & & 05 & 2,1 \\
\hline $2^{\circ}$ grau completo & & 10 & 4,3 \\
\hline Superior completo & & 01 & 0,4 \\
\hline Estado conjugal & 99,2 & & \\
\hline Nunca casou & & 04 & 1,6 \\
\hline Casado(a)/morando junto & & 101 & 42,6 \\
\hline Viúvo (a) & & 99 & 41,8 \\
\hline Divorciado(a)/separado & & 33 & 13,9 \\
\hline Renda mensal & 95,0 & & \\
\hline 1 salário & & 153 & 67,4 \\
\hline Entre 1 e 3 salários & & 65 & 28,6 \\
\hline Maior que 4 salários & & 04 & 1,8 \\
\hline Sem rendimento & & 05 & 2,2 \\
\hline Fonte de renda & 98,3 & & \\
\hline Trabalho & & 28 & 11,7 \\
\hline Pensão ou aposentadoria & & 185 & 87,4 \\
\hline Investimentos & & 27 & 11,3 \\
\hline Dinheiro do cônjuge & & 47 & 19,7 \\
\hline $\begin{array}{l}\text { Dinheiro dos filhos } \\
\text { Dinheiro de amigos/familiares }\end{array}$ & & $\begin{array}{l}75 \\
03\end{array}$ & $\begin{array}{l}31,4 \\
1,3\end{array}$ \\
\hline Outras fontes & & 01 & 0,4 \\
\hline Tipo de moradia & 98,3 & & \\
\hline $\begin{array}{l}\text { Propriedade da } \\
\text { entrevistada ou do casal }\end{array}$ & & 203 & 86,4 \\
\hline $\begin{array}{l}\text { Propriedade do cônjuge do(a) } \\
\text { entrevistado(a) }\end{array}$ & & 03 & 1,3 \\
\hline Alugado pelo entrevistado & & 09 & 3,8 \\
\hline $\begin{array}{l}\text { Morando em residência sem custo } \\
\text { para o entrevistado }\end{array}$ & & 15 & 6,4 \\
\hline Outra categoria & & 05 & 2,1 \\
\hline $\begin{array}{l}\text { Situação econômica comparação } \\
\text { quando tinha } 50 \text { anos }\end{array}$ & 98,3 & & \\
\hline Melhor & & 100 & 42,6 \\
\hline A mesma & & 67 & 28,5 \\
\hline Pior & & 68 & 28,9 \\
\hline $\begin{array}{l}\text { Renda suficiente para suprir as } \\
\text { necessidades }\end{array}$ & 98,3 & & \\
\hline Dá e sobra & & 18 & 7,7 \\
\hline Dá na conta certa & & 88 & 37,4 \\
\hline Sempre falta um pouco & & 98 & 41,7 \\
\hline Sempre falta muito & & 31 & 13,2 \\
\hline
\end{tabular}

Fonte: Dados da pesquisa 
Tabela 2. Arranjo familiar de idosos em estado de corresidência. Vitória da Conquista - BA, 2018.

\begin{tabular}{llll} 
Variáveis & \% resposta & $\mathbf{N}$ & \% \\
\hline Composição do arranjo familiar & 100 & & \\
Esposo(a)/companheiro(a) & & 97 & 40,6 \\
Pais & & 05 & 2,1 \\
Filhos & 132 & 55,2 \\
Filhas & 129 & 54,0 \\
Irmãos/irmãs & 06 & 2,5 \\
Netos(as) & 137 & 57,3 \\
Outros parentes & 16 & 6,7 \\
Outras pessoas & 13 & 5,4
\end{tabular}

\section{Discussão}

Quanto as características sociodemográficas, constatou-se que, dentre os idosos em corresidência houve predominância do sexo feminino, resultado semelhante ao estudo conduzido por Rabelo e Neri ${ }^{5}$, onde $77,6 \%$ dos idosos eram mulheres. Estudos analisam que as mulheres percebem mais facilmente o risco de danos à saúde, favorecendo o diagnóstico e tratamento precoce. Sendo assim, aumenta-se a longevidade com uma melhor condição de saúde.

Ao analisar o valor da renda mensal dos idosos do presente estudo, 67,4\% alegaram receber apenas 01 salário mínimo. Estes achados são corroborados com o estudo de Santos e Pavarini $^{9}$ realizado em 2009 , onde $69 \%$ dos idosos do estudo recebiam de meio a um salário mínimo, e destes, $64 \%$ eram os principais responsáveis pela renda familiar. Em outro estudo coordenado por Bertuzzi, Paskulin e de Morais $^{10}$ dos 36 idosos participantes, mais da metade recebiam um salário mínimo, e em um estudo mais recente realizado por Elias, Marzola e Molina et al $^{11}$ com 637 idosos, verificou-se que, destes, $41,5 \%$ possuíam renda mensal de 1 salário mínimo.

Após os autores Melo, Teixeira e Barbosa ${ }^{12}$ terem realizado um estudo sobre os arranjos familiares no Brasil, foi verificado que a renda salarial para homens idosos era significativamente maior em relação as idosas. A partir dessa reflexão pode-se também atribuir ao fato de que, neste presente estudo, um maior número de mulheres idosas pode influenciar nesse resultado de menor renda mensal, contribuindo com a veracidade de que, na atual conjuntura o salário de homens e mulheres são desiguais, mesmo que em algumas situações ela realize a mesma função do homem.

O idoso no Brasil possui uma situação socioeconômica precária e muitas vezes isso está relacionado ao baixo nível educacional ${ }^{9}$. Neste estudo, a grande parcela dos idosos possuíam o nível primário incompleto, seguido de analfabetos, à vista disso, a literatura atribui que o nível de escolaridade mais baixo influencia diretamente nas condições financeiras do indivíduo, sendo que, quanto mais anos dedicados ao estudo, maiores são as chances de ter um poder aquisitivo melhor, bem como uma melhor renda salarial ${ }^{13,14}$.

Em 2009, Camarano ${ }^{15}$ apresentou que mais da metade $(69,2 \%)$ do orçamento familiar dependia da renda dos idosos, visto que estes, estão vivendo mais e suas condições financeiras estão associadas ao benefício social da aposentadoria. A presença do idoso no domicilio garante uma estabilidade financeira a seus descendentes, mesmo sendo a renda do idoso inferior $^{3}$.

No que concerne às questões sobre a fonte de renda dos idosos em corresidência do presente estudo, verificou-se que grande parte da renda é proveniente de pensões ou aposentadorias. Tal constatação é compatível com o estudo realizado por Meira, Vilela e Casotti et $\mathrm{al}^{2}$, onde $81 \%$ dos entrevistados declararam a aposentadoria como a principal fonte de renda financeira, sendo este, o único recurso para assegurar as necessidades básicas do idoso, que, apesar de dispor de uma renda escassa, ainda contribui financeiramente para a corresidência, incluindo gastos com medicamentos, alimentação, transporte e outros custeios habitacionais. A respeito disso, Camarano et al $^{1}$ ratifica que esses benefícios previdenciários são de extrema importância para a composição da renda dessa família corresidente.

Neste contexto, Meira ${ }^{16}$ destaca que a aposentadoria permite sobremaneira a formação deste arranjo familiar, de forma que este benefício aumenta o poder aquisitivo da população idosa, e em controvérsia, a população mais jovem torna-se dependente 
financeiramente do aposentado. Porém, é necessário pontuar que a grande maioria das pessoas idosas que possui renda igual ou inferior a um salário mínimo, gasta em torno de um quarto da renda com medicamentos, o que interfere diretamente no orçamento.

Tendo a renda da pessoa idosa como um dos principais fatores determinantes para a corresidência, autores como Paulo, Wajnman e de Oliveira $^{17}$ elucidam que a renda do idoso permite que este, tenha autonomia para decidir entre a possibilidade de morar sozinho e preservar sua independência financeira e privacidade, porém essa renda pode atrair os familiares, principalmente os mais desprovidos financeiramente, a coabitarem.

Quanto às questões referentes ao tipo de moradia, uma grande parcela dos idosos declararam que a propriedade em que residiam era da pessoa entrevistada ou do casal, da mesma forma, o estudo conduzido por Meira, Vilela e Casotti et $\mathrm{al}^{2}$ evidenciou que $80,5 \%$ dos idosos declararam ser os proprietários do domicílio em que residia. A este dado, atribui-se que, o fato de os idosos possuírem uma residência fixa também torna a corresidência atrativa para os familiares e reforça a importância do idoso na estrutura de seus descendentes.

No que se refere a percepção de suficiência de renda pelos idosos, $41,7 \%$ destes relataram que sempre falta um pouco de dinheiro para suprir suas necessidades, seguido de $37,4 \%$ que disseram que seus recursos financeiros dá na conta certa, assemelhando-se aos resultados do presente estudo, Meira, Vilela e Casotti et al $^{18}$ observaram que $34,8 \%$ dos idosos apontaram que a renda é capaz de suprir as necessidades de uma forma que dá na conta certa, porém essa mesma pesquisa realizada pelos autores contradiz outro dado do presente estudo ao analisar que apenas $23,7 \%$ dos idosos relataram que sempre falta um pouco para suprir todas as necessidades.

Neste caso, é possível que o convívio intergeracional sobrecarregue a distribuição de renda do idosos, que comumente necessita sustentar toda a família, sendo assim o idoso fica em desvantagem, visto que é esperado, neste momento da vida, que o idoso invista esse dinheiro com seu bem estar próprio, saúde, viagens, lazer, alimentação, vestimenta, entre outros. E por outro lado, o que vemos é a pessoa idosa priorizando as necessidades dos familiares a frente das suas. Inclusive, Melo, Teixeira e Barbosa $^{12}$ pontuam que a desigualdade na distribuição da renda da pessoa idosa, impacta na aquisição de bens de consumo, já que este, com uma renda maior tem melhor poder aquisitivo no que tange compras e serviços.

Embora com uma baixa renda familiar, $42,6 \%$ dos idosos apontaram uma melhor situação econômica em comparação quando tinha 50 anos. Neste contexto, analisa-se que a aposentadoria garante uma maior estabilidade financeira aos idosos, como apresenta Camarano et al $^{1}$ que atribui a influência da renda do idoso no orçamento familiar ao crescimento do benefício social dessa população. Tal constatação é compatível com o estudo de Areosa ${ }^{20}$ onde o autor destaca que, a aposentadoria tem se tornado, gradativamente, a única fonte de renda de inúmeras famílias brasileiras.

No que tange a composição do arranjo familiar observou-se que a maioria dos idosos vivem com netos, seguido por filhos e filhas. Esses resultados reforçam os dados encontrados nos estudos de Meira, Vilela e Casotti et $\mathrm{al}^{18}$ onde os netos (as) representando 71\%, corresponderam a maior parcela da geração que corresidia com o idoso, inverso a isso, no estudo conduzido por Santos e Pavarini ${ }^{8}$ houve mais filhos (56\%) do que netos (31\%) corresidindo com o idoso.

Alguns dos fatores que contribuem para esse resultado pode ser explicado pela pesquisa de Aguiar, Menezes e Camargo ${ }^{19}$, onde as autoras denotam que os idosos na condição de avós tornam-se a principal rede de apoio à família e de cuidados com os netos, principalmente em situações onde os pais encontram dificuldades para conciliar os compromissos profissionais com a criação dos filhos ou até mesmo quando estes se mostram ausentes ou negligentes. Porém, nem sempre os avós têm condição suficiente para o sustento e educação dos netos, visto que ter de arcar com as despesas destes, causa impacto na vida financeira destas pessoas, tendo que redistribuir sua renda.

Camarano et $\mathrm{al}^{1}$ salienta que, a corresidência comumente se dá, em decorrência da necessidade dos filhos ou de outros familiares, já que estes estão dedicando um tempo cada vez maior aos estudos e também devido à instabilidade do mercado de trabalho ou nas relações afetivas. A perda do emprego e divórcios provocam a necessidade de retorno a casa dos pais idosos.

Segundo a literatura, outros fatores que ocasionam a coabitação podem ser citados, como por exemplo a necessidade dos familiares em cuidar do idoso, dessa forma a corresidência lhes possibilitam acompanhar o estado de saúde e 
oferecer cuidado, assim, também evitam a solidão da pessoa idosa, já que a condição de morar sozinho pode levar ao idoso a vivenciar e enfrentar diversas situações e sentimentos, principalmente após a perda/falecimento do cônjuge, dessa forma, a corresidência proporciona amparo, cuidado e evita o isolamento social da pessoa idosa ${ }^{14,21}$.

\section{Conclusão}

O presente estudo obteve através dos resultados, importantes informações sobre os idosos que vivem em corresidência, demonstrando que grande parcela do público estudado possuía apenas um salário mínimo e que a única fonte de renda advém da aposentadoria ou pensão. Também foi possível notar que, quanto a suficiência de renda para as necessidades básicas dos idosos, verificou-se que sempre faltava um pouco, a isso podemos atribuir ao fato de que o idoso corresidente é, em sua grande maioria, o responsável pelo sustento de seus descendentes.

É importante ressaltar que, o presente estudo observou o arranjo familiar mais frequente de idosos corresidindo com netos. Mediante a compreensão da corresidência, este estudo contribui para permitir um maior conhecimento da população e da comunidade cientifica sobre a temática, favorecendo assim, políticas sociais e de saúde para idosos e familiares no intuito de favorecer um bom convívio, uma vez que o próprio estatuto do idoso favorece e estimula o convívio familiar. Por fim, sugere-se a realização de outros estudos com maiores amostras e/ou populações a fim de buscar um maior aprofundamento a respeito da temática e incentivar a explorar de outras formas o universo do envelhecimento e suas especificidades que envolvem o idoso e a família.

\section{Referências}

1. CAMARANO, Ana Amélia et al. Famílias: espaço de compartilhamento de recursos e vulnerabilidades. Os novos idosos brasileiros: muito além dos, v. 60, n. 1, p. 137-167, 2004.

2. MEIRA, Saulo Sacramento et al. Idosos em estado de corresidência em um município do interior da Bahia. Mundo saúde (Impr.), v. 39, n. 2, p. [201-209], 2015.

3. SAMPAIO, Talita Santos Oliveira; SAMPAIO, Lucas Silveira; VILELA, Alba Benemérita Alves. Conteúdos e estrutura representacional sobre família para idosos em corresidência. Ciência \& Saúde Coletiva, v. 24, p.
1309-1316, 2019.

4. BRITO, Jamile dos Anjos, et al. Financial Income and Life Satisfaction of the Elderly Intergenerational Relations. International Journal of Advanced Engineering Research and Science, 6(12), 2019.

5. RABELO, Doris Firmino; NERI, Anita Liberalesso. Tipos de configuração familiar e condições de saúde física e psicológica em idosos. Cadernos de saúde pública, v. 31, p. 874884, 2015.

6. DE PAULA, Flávia Viana et al. Avós e netos no século XXI: autoridade, afeto e medo. Revista da Rede de Enfermagem do Nordeste, v. 12, p. 913-921, 2011.

7. BRASIL. Ministério da Saúde. Secretaria de Atenção à Saúde. Departamento de Atenção Básica. Caderno de Atenção Básica n. 19 Envelhecimento e Saúde da pessoa idosa. Brasília: Ministério da Saúde, 2007.

8. VERAS, Renato; DUTRA, Sidney. Perfil do idoso brasileiro: questionário BOAS. In: Perfil do idoso brasileiro: questionário BOAS. 2008. p. 9898.

9. SANTOS, Ariene Angelini dos; PAVARINI, Sofia Cristina lost. O genograma para caracterizar a estrutura familiar de idosos com alterações cognitivas em contextos de pobreza. Revista Mineira de enfermagem, v. 13, n. 4, p. 525-533, 2009.

10. BERTUZZI, Daiane; PASKULIN, Lisiane Girardi Manganelli; MORAIS, Eliane Pinheiro de. Arranjos e rede de apoio familiar de idosos que vivem em uma área rural. Texto \& ContextoEnfermagem, v. 21, n. 1, p. 158-166, 2012.

11. ELIAS, Henrique Ciabotti et al. Relação entre funcionalidade familiar e arranjo domiciliar de idosos. Rev Bras Geriatr Gerontol [Internet], v. 21, n. 5, p. 582-90, 2018.

12. DE MELO, Natália Calais Vaz et al. Arranjo domiciliar de idosos no Brasil: análises a partir da Pesquisa Nacional por Amostra de Domicílios (2009). Revista Brasileira de Geriatria e Gerontologia, v. 19, n. 1, p. 139-151, 2016

13. SOARES, llana da Silva, et al. Elderly health conditions in coresidence. International Journal of Development Research, vol 08, edição 06, pp.21168-21173,2018.

14. MELO, N. C. V.; FERREIRA, M. A. M.; TEIXEIRA, K. M. D. Condições de vida dos idosos no Brasil: uma análise a partir da renda e nível de escolaridade. Oikos: Rev Bras Econ Doméstica. 2014 [cited 2017 Aug 25]; 25 (1): 4-19

15. CAMARANO, Ana Amélia. Relações familiares, trabalho e renda entre idosos. Barros Júnior JC, organizador. Empreendedorismo, trabalho e qualidade de vida na terceira idade. 
São Paulo: Edicon, p. 81-96, 2009

16. MEIRA, SAULO SACRAMENTO. Estudo de base populacional entre as condições sociais $\mathrm{e}$ autoestima de idosos mineiros corresidentes. Dissertação [Mestrado]. Programa de PósGraduação em Enfermagem e Saúde, Universidade Estadual do Sudoeste da Bahia, Jequié, BA. 2013. 98 p.

17. PAULO, Maira Andrade; WAJNMAN, Simone; DE OLIVEIRA, Ana Maria Camilo Hermeto. A relação entre renda e composição domiciliar dos idosos no Brasil: um estudo sobre o impacto do recebimento do Benefício de Prestação Continuada. Revista Brasileira de Estudos de População, v. 30, p. S25-S43, 2013.

18. MEIRA, Saulo Sacramento et al. Considerações acerca das condições de vida de idosos no Vale do Jequitinhonha, Minas Gerais, Brasil. Serviço Social em Revista, v. 17, n. 1, p. 159-177, 2014.

19. AGUIAR, Aline Cristiane de Souza Azevedo; DE OLIVA MENEZES, Tânia Maria; DE CAMARGO, Climene Laura. Arranjos familiares com pessoas idosas: fatores contributivos. Avances en Enfermería, v. 36, n. 3, p. 292-301, 2018.

20. AREOSA, Silvia Virginia Coutinho. Idosos Provedores: a importância dos recursos da aposentadoria para as famílias brasileiras. Barbarói, p. 173-184, 2015.

21. DA CUNHA, Ubiracelma Carneiro; DIAS, Cristina Maria de Souza Brito. A recoabitação dos filhos e netos na perspectiva de idosas chefes de família. Contextos Clínicos, v. 12, n. 2, p. 599-616, 2019.

\section{Endereço para Correspondência}

Faculdade Independente do Nordeste -FAINOR

Av. Luis Eduardo Magalhães, 1305 - Candeias

Vitória da Conquista (BA)

e-mail: jainneleao2015@gmail.com

Recebido em 17/11/2020

Aprovado em 27/02/2021

Publicado em 31/03/2021 